\title{
Time dependent evaluation of the lightning upward connecting leader inception
}

\author{
Marley Becerra and Vernon Cooray \\ Division for Electricity and Lightning Research, Uppsala University, Sweden and \\ The Ångström Laboratory, Box 534, SE 751 21, Uppsala, Sweden \\ E-mail: Marley.Becerra@angstrom.uu.se
}

Received 21 May 2006

Published 20 October 2006

Online at stacks.iop.org/JPhysD/39/4695

\begin{abstract}
The evaluation of the upward connecting leader inception from a grounded structure has generally been performed neglecting the effect of the propagation of the downward stepped leader. Nevertheless, field observations suggest that the space charge produced by streamer corona and aborted upward leaders during the approach of the downward lightning leader can influence significantly the initiation of stable upward positive leaders. Thus, a physical leader inception model is developed, which takes into account the electric field variations produced by the descending leader during the process of inception. Also, it accounts for the shielding effect produced by streamer corona and unstable leaders formed before the stable leader inception takes place. The model is validated by comparing its predictions with the results obtained in long gap experiments and in an altitude triggered lightning experiment. The model is then used to estimate the leader inception conditions for free standing rods as a function of tip radius and height. It is found that the rod radius slightly affects the height of the downward leader tip necessary to initiate upward leaders. Only an improvement of about $10 \%$ on the lightning attractiveness can be reached by using lightning rods with an optimum radius. Based on the obtained results, the field observations of competing lightning rods are explained.

Furthermore, the influence of the average stepped leader velocity on the inception of positive upward leaders is evaluated. The results obtained show that the rate of change of the background electric field produced by a downward leader descent largely influences the conditions necessary for upward leader initiation. Estimations of the leader inception conditions for the upper and lower limit of the measured values of the average downward lightning leader velocity differ by more than $80 \%$. In addition, the striking distances calculated taking into account the temporal change of the background field are significantly larger than the ones obtained assuming a static downward leader field. The estimations of the present model are also compared with the existing leader inception models and discussed.
\end{abstract}

(Some figures in this article are in colour only in the electronic version)

\section{Introduction}

From experiments in long sparks [1-3] it has been found that the conditions necessary for the inception of stable (i.e. propagating) leaders depend upon several factors. The most influential of these factors include the geometry of the configuration and the manner in which the applied electric fields change in time. Thus, the models proposed 
to estimate the leader initiation condition are valid for a given configuration and a particular voltage waveshape. The critical radius concept introduced by Carrara and Thione [4] was obtained for sphere to plane and conductor to plane configurations under switching impulse voltages $230 / 3000 \mu \mathrm{s}$. However, different critical radii have been obtained for other waveshapes [3] even for the same configuration. The generalized leader inception criterion proposed by Rizk $[5,6]$ was derived under the critical condition when the instantaneous applied impulse voltage increases so as to maintain a sensibly constant leader tip potential as the leader continues to penetrate the gap' [5]. This condition is satisfied in long gaps for voltages in which the critical time to crest is about $500 \mu \mathrm{s}$ [3]. Later, in order to simulate the leader process under any voltage waveform, more sophisticated physical models were proposed [7-9] to evaluate the leader inception as well as its propagation.

Due to various similarities of the upward positive leaders initiated from grounded structures under the influence of downward lightning stepped leaders and those that occur in long sparks, the models mentioned above have been applied to the case of natural lightning. For example, the critical radius concept is used as a leader inception criterion in lightning related studies by Eriksson [10], Dellera and Garbagnati [11] and D'Alessandro [12]. The critical radius used is $0.36 \mathrm{~m}$ obtained by Carrara and Thione [4] and $0.28 \mathrm{~m}$ obtained by Bernardi et al [13] for a grounded rod between two parallel plates with a 500/6000 $\mu$ s waveform. In addition, Rizk [14] developed a lightning model based on his leader inception model that is applied to the analysis of transmission lines, rods and masts.

Based on the experimental data obtained in long gaps, other models have also been developed for the initiation of upward leaders from grounded structures. Petrov and Waters [15] proposed a leader inception criterion based on the length of the streamer zone and the range of the electric field intensification. According to this model, when the streamers extend beyond a critical length equal to $0.7 \mathrm{~m}$, an upward leader is initiated. However, this critical length is observed to depend upon the steepness of the applied fields [3] and it is also affected by the space charge produced by streamers before inception. This model was extended in [16] by considering that the stable propagation of the incepted leader is reached if the rate of change of the induced potential by the downward leader at the rod's tip is larger than $6 \mathrm{kV}_{\mu \mathrm{s}}{ }^{-1}$. Bazelyan and Raizer [17] proposed a semiempirical leader model based on laboratory data and applied it to study the lightning strikes to free standing masts. But it is not clear whether the laboratory based empirical values of this model can be applied to the actual case of inception in the presence of a downward lightning leader.

Lalande [18] used the physical model for the leader propagation in long gaps proposed in [9] to derive an equation to compute the leader inception fields under static condition. In other words, it was assumed that the electric field produced by the downward leader does not vary during the inception process. Such a condition is valid only for the inception of leaders from tall objects under the influence of the electric fields produced by thunderclouds and for the inception of upward leaders in rocket triggered lightning. Later, Becerra and Cooray [19] using similar physics derived a leader inception model to describe the inception and propagation of the first few metres of an upward leader from structures with and without axial symmetry. Similar to Lalande [18] they assumed static conditions in their model.

As one can observe, some of the leader inception models described above are based on experimental and empirical relationships derived from laboratory experimental results. The other models which utilize the physics of air breakdown assume static conditions during the leader inception and propagation. Nonetheless, there are some physical facts that cast doubt on the validity of these leader inception models when they are used to describe the launching of upward leaders from grounded structures under the influence of a downward moving stepped leader. First of all, the temporal change of the electric field produced by the downward leader at the point of initiation of the upward leader is different from the time variation of the electric fields applied in the laboratory. The rate of change of the background electric field produced by a downward moving leader increases exponentially and it can reach values higher than $10 \mathrm{kV} \mathrm{m}^{-1} \mathrm{~ms}^{-1}$ when the leader is close to the ground [20,21]. Whereas the inception and the propagation of the leaders in most of the laboratory experiments take place when the rate of change of the applied electric field is decreasing [16]. Moreover, the rate of change of the background electric fields in long gap experiments reach values larger than $300 \mathrm{kV} \mathrm{m}^{-1} \mathrm{~ms}^{-1}$ [13]. Thus, the initiation of upward positive leaders from grounded structures during the descent of the downward leader cannot be analysed either by using the static approach or by appealing directly to the experimental data from the long spark experiments.

Another difference between the natural situation and the laboratory experiments is the following. As the lightning stepped leader approaches to ground, several bursts of streamers and aborted leaders are produced from grounded rods before the inception of the stable upward leader. This fact is not observed in laboratory experiments [1-3] but it has been found in field measurements associated with lightning flashes [22,23]. The charge associated with these streamer bursts and aborted leaders reduce the electric field in front of the rod. This shielding effect inhibits the development of subsequent corona and the inception of a stable leader, as it has been seen in triggered lightning experiments [21,24].

Hence, to properly evaluate the initiation of upward connecting positive leaders under the influence of downward stepped leaders, one has to develop a model based on physics that can accommodate the above described effects. Since the model introduced by Becerra and Cooray [19] can include the effects of space charge, it is extended in this paper to account also for the dynamic variation of the electric field produced by the downward leader. In this way, the upward positive leader inception from grounded structures can be evaluated under the dynamic conditions imposed by the descent of the downward stepped leader. These conditions include the time variation of the electric field produced by the stepped leaders as well as the space charge associated with streamers and aborted leaders generated before the stable leader inception takes place. The resulting model is validated by comparing its predictions with long gap experiments and an altitude triggered lightning experiment. In this paper we apply that model also to evaluate 

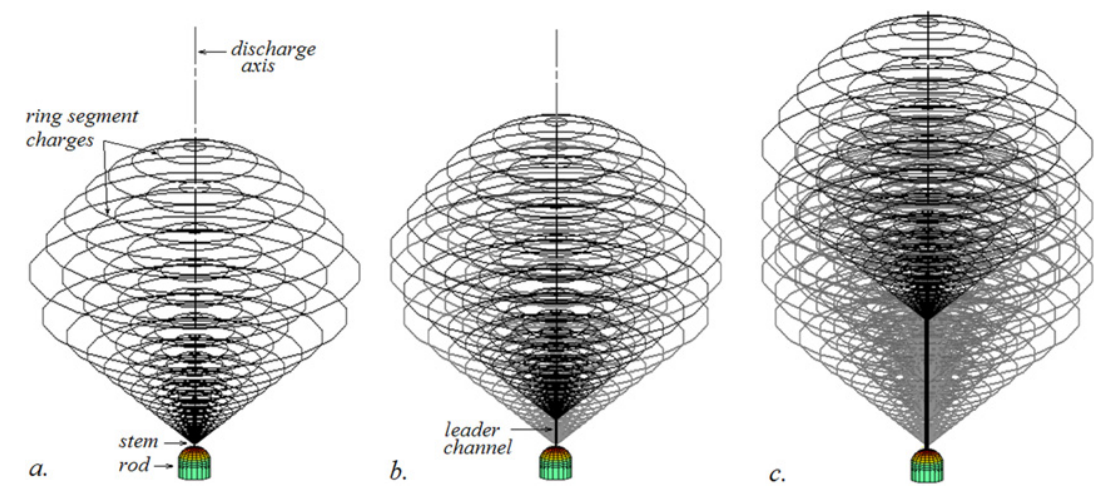

Figure 1. CSM modelling of the upward leader propagation: $(a)$ first corona ring charges, $(b)$ and $(c)$ corona zone charges in front of the leader channel as the leader propagates in the presence of the previous space charge around the discharge axis.

the inception of upward connecting positive leaders when the downward stepped leader approaches to grounded rods.

\section{Physical positive upward leader inception model}

Let us first briefly describe the original model of Becerra and Cooray [19]. It assumes the electrostatic representation of the corona zone proposed by Goelian et al [9] which considers that this zone is characterized by a constant potential gradient $E_{\text {str }}$. Then, the charge simulation method (CSM) [25] is used to compute the total corona charge required to satisfy this condition. The stem/leader channel is represented by finite lines with increasing length and a point charge at its tip while the corona streamer zone is modelled by using generalized ring segment charges of uniform charge density as shown in figure 1. The detailed explanation of the CSM calculation can be found in [19].

The simulation starts by computing the height of the downward leader tip where streamers are incepted from the analysed rod. The well-known streamer criterion [26] is used assuming that enough free electrons are always available to start the discharge. Hence, any statistical time lag is neglected in the evaluation of the streamer inception condition. This assumption is justified since glow corona is usually produced from grounded rods before the streamer inception [27], which provides enough free electrons. In addition, any delay in the streamer inception produced by the injected glow corona charge during the thundercloud charging and the descent of the downward leader [27] is neglected.

The model first simulates the corona zone of the first streamer burst. Due to the fact that the streamer corona charge shields the electric field at the rod tip, a dark period occurs [26] where no streamers are produced. The dark period ends when the streamer inception condition is satisfied again by the increase of the electric field caused by the descent of the downward leader. For the sake of simplicity, the movement of positive ions is neglected in the calculation.

This analysis is repeated until the total charge $\Delta Q$ of any subsequent corona burst is equal to or larger than $1 \mu \mathrm{C}$ [26]. This value corresponds to the critical charge required to thermalize the stem of the corona discharge, creating the first leader segment. This condition defines the transition from streamer to leader, known as the unstable leader inception.
Since this condition is not sufficient to guarantee the selfpropagation of the leader (stable leader inception), the leader advancement is simulated as in [28], but only considering the first few metres of leader propagation.

It is important to point out that the presented model can be used to simulate the behaviour of the leaders in either laboratory sparks or lightning. Since it is based on the physics of discharges, the leader inception can adequately be evaluated provided that the correct background field is used as input. Let us consider the analysis for both cases in the next section.

\section{Validation of the leader inception model under changing electric fields}

In order to test the validity of the model when the time variation of the background electric fields is incorporated, two laboratory experiments [1-3] with $10 \mathrm{~m}$ long sparks are analysed. Thus, the simulations are started at the time $T_{\mathrm{i}}$ at which the first corona is observed in the experiment, corresponding to an applied voltage equal to $U_{\mathrm{i}}$. Table 1 shows the computed time and voltage when the unstable $\left(T_{\mathrm{i}}^{\prime}\right.$ and $\left.U_{\mathrm{i}}^{\prime}\right)$ and stable $\left(T_{1}\right.$ and $\left.U_{1}\right)$ leaders are initiated. For comparison purposes the measured values of these parameters are also given in table 1 . The time of stable leader inception is obtained from the extrapolation of the leader tip position back along its continuous growth from the streak image, as shown in figure 2. This is the way in which these times are estimated in the laboratory experiments [3]. Note the good agreement between the measured and computed leader inception time.

Now, let us apply the model to a lightning environment. In this case, the experimental data obtained in the altitude triggered lightning experiment 9516 reported in [21] is utilized. In altitude triggering, the effect of a downward stepped leader is reproduced to some extent by using two metal wires connected to each other by an insulating string. The length of the insulating string can be several hundred metres. In this technique a positive leader is initiated from the top end of the upper floating wire and consequently a negative leader is initiated from its bottom end. In response to this downward moving negative leader, an upward moving positive leader is initiated from the grounded bottom wire section. The model is utilized to predict the stable leader inception from the grounded wire. 
Table 1. Measured (M) and computed (C) conditions for inception of unstable and stable leaders in two long gap experiments.

\begin{tabular}{|c|c|c|c|c|c|c|c|c|c|}
\hline \multicolumn{2}{|c|}{ Corona } & \multicolumn{4}{|c|}{ Unstable leader } & \multicolumn{4}{|c|}{ Stable leader } \\
\hline \multirow{2}{*}{$\begin{array}{l}T_{\mathrm{i}}[\mu \mathrm{s}] \\
\mathrm{M}\end{array}$} & \multirow{2}{*}{$\begin{array}{l}U_{\mathrm{i}}[\mathrm{kV}] \\
\mathrm{M}\end{array}$} & \multicolumn{2}{|c|}{$T_{\mathrm{i}}^{\prime}[\mu \mathrm{s}]$} & \multicolumn{2}{|c|}{$U_{\mathrm{i}}^{\prime}[\mathrm{kV}]$} & \multicolumn{2}{|c|}{$T_{1}[\mu \mathrm{s}]$} & \multicolumn{2}{|c|}{$U_{1}[\mathrm{kV}]$} \\
\hline & & M & $\mathrm{C}$ & M & $\mathrm{C}$ & M & $\mathrm{C}$ & M & $\mathrm{C}$ \\
\hline \multicolumn{10}{|c|}{ Hemisphere $0.1 \mathrm{~m}$ diameter, $10 \mathrm{~m}$ gap, $1890 \mathrm{kV}, 600 / 10000 \mu \mathrm{s}[3]$} \\
\hline $42 \pm 6$ & $504 \pm 70$ & $64=$ & 74 & 720 & 810 & $97 \pm 25$ & 86 & $977 \pm 180$ & 907 \\
\hline \multicolumn{10}{|c|}{ Cone 0.01 tip radius, $10 \mathrm{~m}$ gap, $1850 \mathrm{kV}, 500 / 3800 \mu \mathrm{s}[1,2]$} \\
\hline $21 \pm 4$ & $243 \pm 50$ & - & 35 & - & 579 & $\sim 45$ & 47 & $\sim 721$ & 736 \\
\hline
\end{tabular}

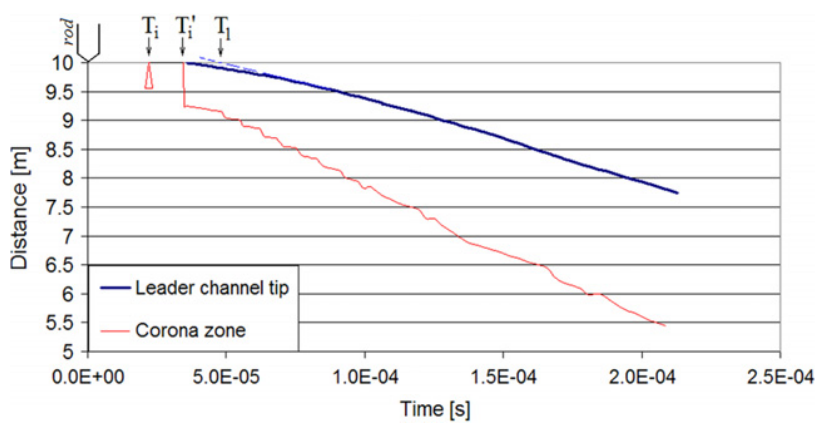

Figure 2. Computed streak image for a $10 \mathrm{~m}$ long cone to plane configuration during the inception and early propagation stage of the leader when a $1850 \mathrm{kV}$ impulse voltage with $500 \mu$ s time to crest is applied.

In this experiment, it was observed that the inception of the upward leader from the grounded wire occurred $4.02 \mathrm{~ms}$ after the bottom of the floating wire reached a height of $450 \mathrm{~m}$. The length of the bottom wire at this time was $50 \mathrm{~m}$ and the insulating string is $400 \mathrm{~m}$ long. In order to simulate these experimental conditions, the grounded wire segment is simulated by a hemispherically capped grounded rod while the downward leader is assumed to be a uniform line charge located above the rod and extending downwards from a point located at $450 \mathrm{~m}$. The average velocity of the downward leader is $1.3 \times 10^{5} \mathrm{~m} \mathrm{~s}^{-1}$ when the leader has extended to a length of about $330 \mathrm{~m}$, according to the value estimated in the experiment [21]. In the simulation, the value of the linear charge density on the negative leader is selected in such a way that the ground electric field produced by the leader at a point located $50 \mathrm{~m}$ away from the wire agrees with the measurements. Since the static background electric field produced by the thundercloud during the experiment was not reported, a typical value of about $15 \mathrm{kV} \mathrm{m}^{-1}$ is taken from the electric field profile measured by Willet et al [24] at an altitude of $50 \mathrm{~m}$.

Figure 3 shows the streak image and the leader current computed with the presented model for the triggered lightning experiment. The reported values in [21] of the electric field at ground level $50 \mathrm{~m}$ away from the wire and the current at the base of the grounded wire are also shown. Since the measured values of the ground electric field before the leader inception (at about $4 \mathrm{~ms}$ ) are not reported in [21], an exponential increase of the electric field is initially assumed. It is found that some leaders are aborted before the inception of the stable leader. The transition of streamer to leader that led to the first aborted leader takes place at $3.68 \mathrm{~ms}$ while the unstable inception of the final self-propagating leader occurred about $32 \mu$ s later.
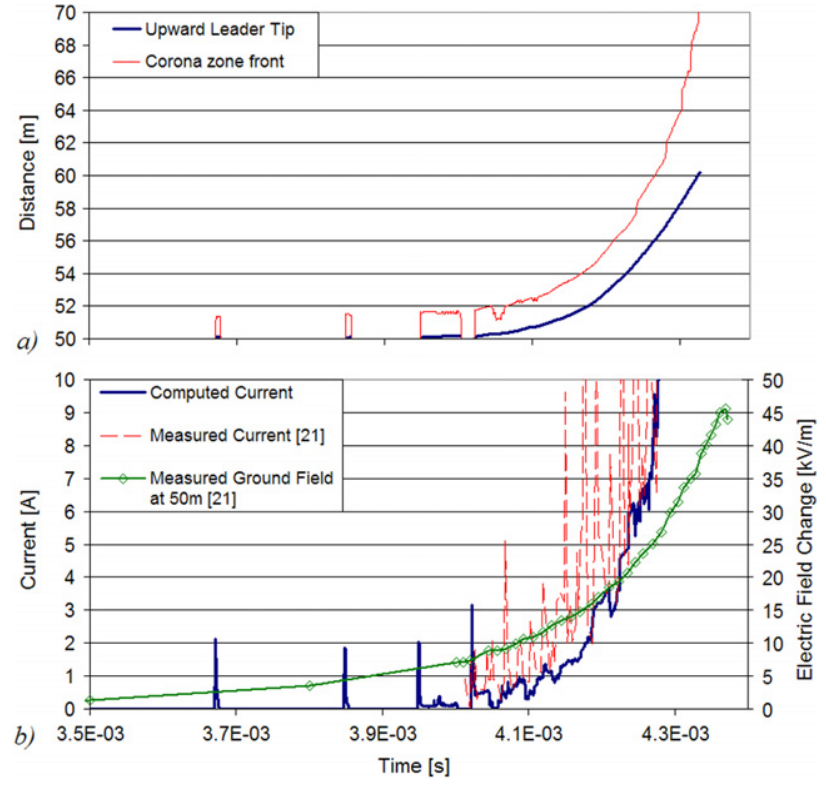

Figure 3. Comparison of the measured [21] and computed conditions during the inception of an upward leader in an altitude triggered lightning experiment: $(a)$ streak image, $(b)$ computed and measured current and field measured at ground level $50 \mathrm{~m}$ away from the wire. The time scale is initiated at the moment when the floating conductor reaches $450 \mathrm{~m}$ above ground.

The upward leader started its continuous propagation around $4 \mathrm{~ms}$, in excellent agreement with the experimentally estimated leader inception time of $4.02 \mathrm{~ms}$ [21]. Furthermore, observe the excellent agreement between the computed current and the main component of the measured current at the base of the grounded wire after the leader inception. However, the isolated pulses on the measured current produced by the discontinuous stepped propagation mode of the upward leader [21] are not reproduced since only the continuous advancement of the leader is considered here.

\section{Dynamic inception of positive upward connecting leaders from grounded rods}

Even though the leader inception model described here can be applied to any kind of structure, in this paper we will confine ourselves to the study of leader inception conditions of grounded isolated rods. Figure 4 shows an example of the model predicted processes taking place during the inception of an upward leader from a $10 \mathrm{~m}$ high rod of $0.05 \mathrm{~m}$ tip radius. A downward leader with prospective return stroke peak current 
Dynamic evaluation of the lightning upward leader inception

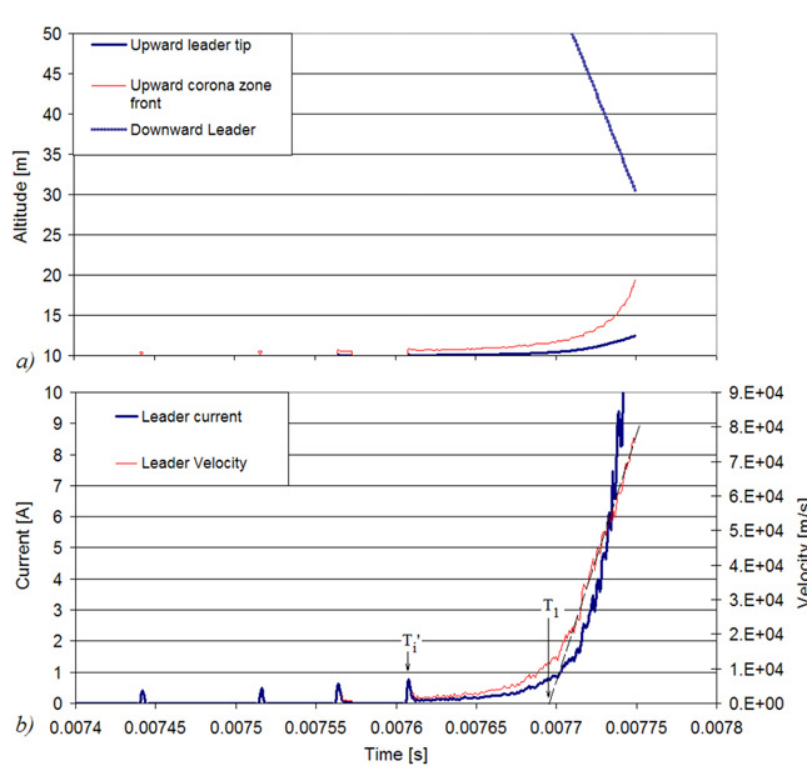

Figure 4. Computed discharge characteristics during leader inception for a $10 \mathrm{~m}$ high, $0.05 \mathrm{~m}$ tip radius rod in the presence of a downward leader with $10 \mathrm{kA}$ prospective return stroke current: $(a)$ streak image, $(b)$ leader velocity and current.

of $10 \mathrm{kA}$, approaching the rod with a continuous velocity of $5 \times$ $10^{5} \mathrm{~m} \mathrm{~s}^{-1}$ (without stepping) is considered. The charge density of the downward leader is computed by using the equation derived by Cooray et al [29]. Observe that before the unstable leader inception time $T_{\mathrm{i}}^{\prime}$, streamers and leaders are produced at the tip of the rod. These aborted discharges produce dark periods until the electric field conditions at the rod tip are restored by the increase on the downward leader background field. Note that the computed currents show similar features than the measured currents during the inception of upward leaders from lightning rods under thunderstorm conditions [22,23] and in triggered rocket experiments [21].

Once the first segment of the successful leader is initiated at $T_{\mathrm{i}}^{\prime}$, its propagation is weak (with low current) and in this particular case it advances less than $1 \mathrm{~m}$ in about $100 \mu \mathrm{s}$. After some time, the electric field produced by the downward leader is high enough to accelerate the leader and start its continuous propagation. Since the extrapolation of the leader tip position back along its continuous growth used in long sparks [3] is found to be inconvenient to estimate the stable inception time of upward connecting leaders, the stable leader inception time $T_{1}$ is defined here as follows. As one can see in figure $4(b)$, at a certain time after the unstable inception takes place, the leader starts to accelerate more or less uniformly. Hence, the time of inception of stable leader is defined as the crossing point of a line that connects the leader velocity between $2 \times 10^{4}$ and $4 \times 10^{4} \mathrm{~m} \mathrm{~s}^{-1}$ and the time axis.

In the simulations, the leader inception times $T_{\mathrm{i}}^{\prime}$ and $T_{1}$ are computed for a $10 \mathrm{~m}$ height rod under the influence of a downward leader, as function of the rod tip radius. A prospective peak return stroke current of $10 \mathrm{kA}$ and an average stepped leader velocity of $5 \times 10^{5} \mathrm{~m} \mathrm{~s}^{-1}$ are assumed. The computed height of the downward leader tip above ground corresponding to the unstable and stable leader inception is shown in figure 5. First of all, observe that the height of the downward leader necessary to incept an unstable leader

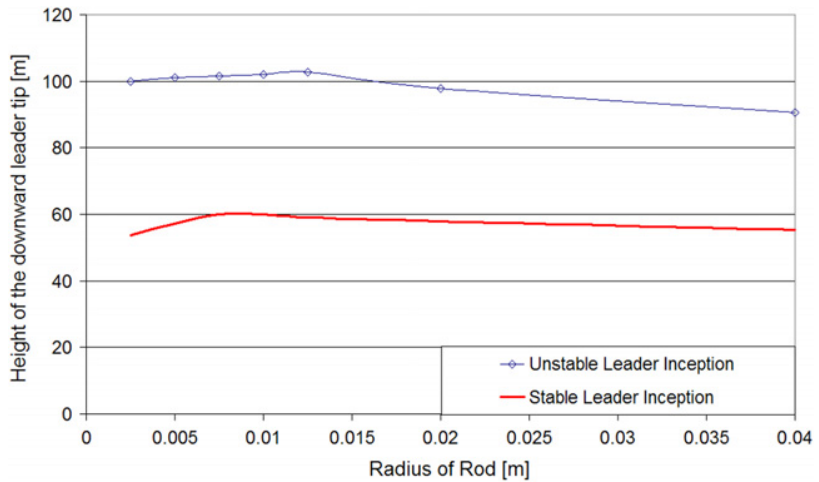

Figure 5. Height of the downward leader tip where unstable and stable upward leader inception takes place from a $10 \mathrm{~m}$ high rod as function of its radius. The downward stepped leader is assumed to have prospective return stroke peak current of $10 \mathrm{kA}$ and average velocity of $5 \times 10^{5} \mathrm{~m} \mathrm{~s}^{-1}$.

is about twice larger than to initiate a self-propagating leader. Also note that the tip radius slightly affects the height of the downward leader where the leader inception takes place. But these differences are not larger than about $10 \%$ for the range of tip radius considered. However, note that there is an optimum rod radius for which the height of the tip of the downward moving stepped leader that can generate a stable (and unstable) upward leader from the rod is maximum. In other words, this rod radius is slightly more efficient than other rod radii in generating both unstable and stable upward leaders. For tip radii lower than the optimum, several bursts of streamers with low charge (lower than $1 \mu \mathrm{C}$ ) are initiated, reducing the charge of the subsequent streamer bursts and retarding the unstable leader inception. On the other side, blunter rods produce less streamer bursts and the dark periods are larger, also retarding the unstable condition. The optimum radius for the stable leader inception is slightly shorter than for the unstable condition because it depends on the most favourable conditions for the leader propagation.

These predicted differences between rods with different radii are in agreement with the field observations of competing $6 \mathrm{~m}$ tall lightning rods in the Magdalena Mountains in New Mexico [22,23]. During a period of seven years, only blunt rods with tip diameters from 12.7 to $25.4 \mathrm{~mm}$ have been struck by lightning while none of the sharp Franklin rods, the 'Early Streamer Devices', or the blunt rods with radii larger than $25.4 \mathrm{~mm}$ received any strike. At first sight, this result suggests that the rods with diameter between 12.7 and $25.4 \mathrm{~mm}$ are more effective to intercept lightning flashes than other rods [22]. However, according to our calculations in figure 5, the rods within that range can be only up to $10 \%$ more efficient than other rods. The question is how this $10 \%$ advantage caused all the lightning flashes to strike the rods with radii in the vicinity of optimum values.

According to the opinion of the authors, the differences between rods found by Moore [22,23] are influenced by the setup of the experiment. Since the competing rods were placed rather close to each other (about $6 \mathrm{~m}$ apart), the small advantage of the 'optimum' rods (figure 5) cause them to initiate upward leaders before the others. Even though the presence of the adjacent rods produced a change of only about $1 \%$ on the electric field at the tip of each rod [22], a much larger shielding 
of the electric field is produced when the corona zone and leader channel is formed from any rod. Thus, once an upward leader is initiated from a rod, it screens the other rods and inhibits the development of stable upward leaders from them. In this way, the 'optimum' rods always received the lightning strikes during the experiment, suggesting that the other rods are not effective strike receptors [22]. Nonetheless, if the rods were competing fully independent from each other, a different conclusion could have been reached.

In addition, it is noteworthy that the 'optimum' rod radii obtained in figure 5 only apply for free standing rods up to about $10 \mathrm{~m}$ height. For taller rods, the differences between sharp and blunt rods on the height of the downward leader required to incept an upward leader become even less significant. This is because the frequency and magnitude of streamer corona generated due to the field enhancement at the tip of the rod becomes less dependent upon its radius as the rod height increases. Hence, the effect of the streamer corona injected during the descent of the downward leader on the conditions for the inception of upward leaders from tall rods is about the same for the range of the rod radius considered in this paper.

\section{Effect of the average velocity of the downward stepped leader in the upward positive leader inception}

As mentioned before, the initiation of stable upward leaders depends also upon the rate of change of the applied electric fields. In the case of natural lightning, the temporal change of the electric fields depends mainly upon the velocity of the downward stepped leader [30]. A larger stepped leader velocity produces a higher temporal change of the electric fields close to ground. Based on the review reported by Rakov and Uman [31], the measured and estimated values of the average stepped leader velocity range from $8 \times 10^{4}$ to $3.9 \times 10^{6} \mathrm{~m} \mathrm{~s}^{-1}$ in the final hundreds of metres above ground. Therefore, in order to evaluate the effect of the stepped leader velocity on the initiation of upward leaders, the proposed leader inception model is applied to a $10 \mathrm{~m}$ high, $0.01 \mathrm{~m}$ tip radius rod. Figure 6 shows the results obtained for a downward leader with $10 \mathrm{kA}$ prospective return stroke current, which is assumed to approach the rod with a continuous average velocity within the range mentioned above. The pulses in the electric field produced by the stepping of the downward leader [32] are neglected for sake of simplicity.

As the stepped leader velocity augments, both the frequency and charge of the corona bursts increase. For this reason, the height of the stepped leader tip required to reach unstable leader inception initially increases with the downward leader velocity (figure 6). However, this increase has an upper limit which corresponds to the maximum height of the stepped leader tip that produces enough electrostatic energy to reach the corona critical charge of $1 \mu \mathrm{C}$. This corona critical charge is reached approximately at the same tip height for stepped leader velocities larger than about $5 \times 10^{5} \mathrm{~m} \mathrm{~s}^{-1}$.

On the other side, the height of the stepped leader tip that initiates a stable upward leader from an isolated rod monotonously increases with increasing average leader velocity (figure 6). This is because an increment of the average stepped leader velocity leads to a larger rate of change

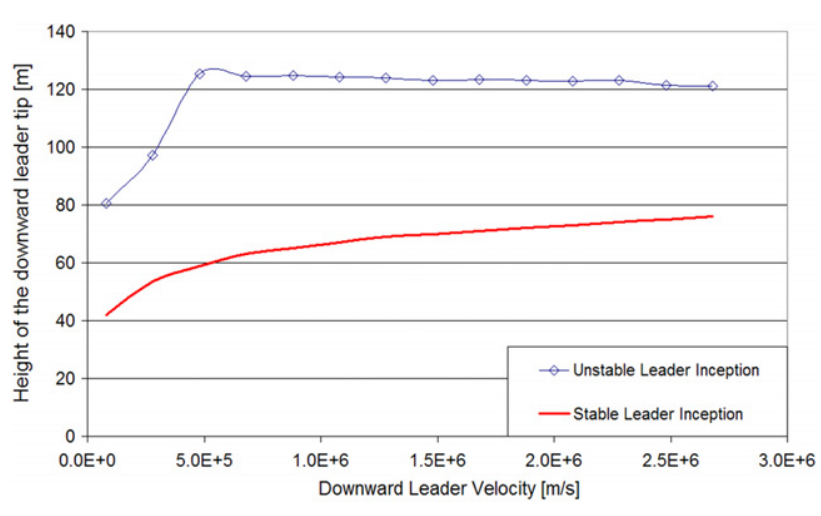

Figure 6. Height of the downward leader tip where unstable and stable upward leader inception takes place from a $10 \mathrm{~m}$ high, $0.01 \mathrm{~m}$ tip radius rod as a function of the average leader velocity. A prospective return stroke peak current of $10 \mathrm{kA}$ is assumed.

of the background electric field. In this way, the available electrostatic energy in front of the upward leader tip becomes large enough to compensate the potential drop of a leader during its propagation. This fact facilitates the formation of corona charge at the leader tip and therefore the creation of a larger new leader segment. Hence, the self-propagation of a positive upward leader and consequently the stable leader inception are largely influenced by the downward stepped leader velocity. As can be seen in figure 6, the tip height of a slow moving downward leader (with velocity of $8 \times 10^{4} \mathrm{~m} \mathrm{~s}^{-1}$ ) that leads to the inception of a stable upward leader is 1.8 times smaller than that for the case of a stepped leader with high average velocity of $2 \times 10^{6} \mathrm{~m} \mathrm{~s}^{-1}$. This fact suggests that the speed of the downward stepped leader is also an important parameter to account for in the analysis of lightning attachment to grounded structures.

\section{Calculation of the effective striking distance}

For the evaluation of lightning interception conditions of grounded structures, the concept of striking distance is generally used. It is defined as the distance between the tip of the downward leader and the rod tip where the inception of a stable upward leader takes place [10]. In order to compare the differences obtained by evaluating the dynamic leader inception under the influence of a downward coming leader and the predictions of the existing models, the striking distances are computed for a $0.01 \mathrm{~m}$ tip radius rod. Figure 7 shows the obtained results as function of the height of the rod for a downward leader with $10 \mathrm{kA}$ prospective return stroke peak current. A mean average downward leader velocity of $2 \times 10^{5} \mathrm{~m} \mathrm{~s}^{-1}$ is assumed and the bars correspond to the striking distance computed for the upper and lower limits of the measured stepped leader velocities (corresponding to $8 \times 10^{4}$ and $3.9 \times 10^{6} \mathrm{~m} \mathrm{~s}^{-1}$ ). The calculations are performed considering a thundercloud electric field of $10 \mathrm{kV} \mathrm{m}^{-1}$ [11]. The striking distance computed with the Becerra and Cooray leader inception model [19] is also evaluated under the presence of the downward leader but assuming a static condition where the background electric field is considered invariant. In addition, the striking distances with the critical radius concept (for critical radius of $0.36 \mathrm{~m}$ 


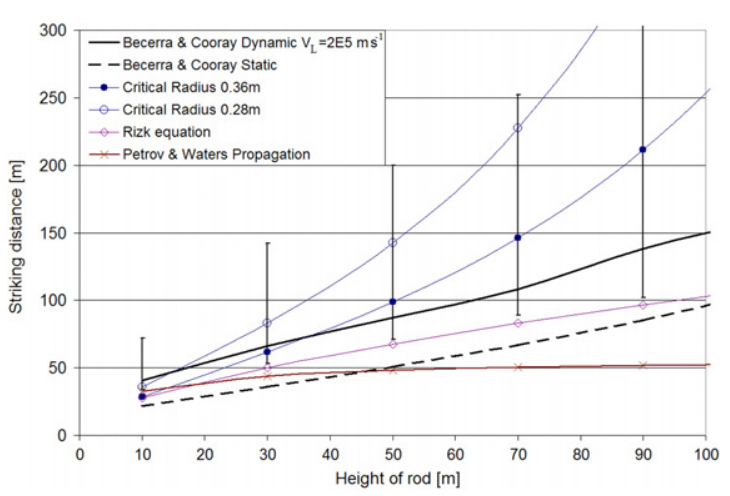

Figure 7. Striking distance of a $0.01 \mathrm{~m}$ tip radius rod as a function of its height. A prospective return stroke peak current of $10 \mathrm{kA}$ is assumed.

[4] and $0.28 \mathrm{~m}$ [13]), the Rizk generalized leader inception equation [5] and the field intensification criterion of Petrov and Waters $[15,16]$ are computed at the downward leader tip height where each criterion is satisfied. The Bazelyan and Raizer's equation $[17,35]$ and the Lalande's stabilization field equation [18] are not evaluated in this paper since they are based on the concept of a stabilization field in static conditions. Even though the striking distance for these two models could be estimated as the height of the downward leader tip that produces an average background field over the height of the rod equal to the stabilization field, this assumption is not valid for structures larger than about $50 \mathrm{~m}$ [19].

First of all, observe that the striking distances computed considering the dynamic upward leader inception process (solid line in figure 7) are significantly larger that those obtained assuming a static condition (dashed solid line in figure 7). Because of the increasing background field produced by the descent of the downward leader, the propagation of an upward connecting leader becomes self-sustained earlier than in the case of an invariant static electric field. Hence, the estimated striking distances significantly depend upon the downward stepped leader velocity, as mentioned in the previous section. Note that the wide range of the striking distances estimated with the present model, shown with bars in figure 7 , is produced by the large spread of the measured values of the downward leader average velocity. Based on this, the static evaluation of the leader inception [19] particularly applies to slowly changing background fields as in the case of upward initiated flashes from tall towers and classical triggered lightning experiments. However, this static approach can be used for comparative studies where the absolute value of the striking distance is not a critical issue, as in [33,34]. Despite the fact that the striking distances computed assuming a static condition [19, 33, 34] are much more conservative than in reality, this approach can be easily implemented to conduct qualitative analyses of lightning interception because of its simplicity. Furthermore, observe that the striking distances computed assuming a static electric field are in some ways proportional to those obtained with the dynamic inception model for a downward velocity of $2 \times 10^{5} \mathrm{~m} \mathrm{~s}^{-1}$.

As to the other leader inception models, note the large differences between the estimations of each model, mainly due to the assumptions considered in each case. Observe that both the critical radius concept and the Rizk equation, originally proposed for the changing electric fields generated in the laboratory predict larger striking distances than the model when a static condition is considered. For very short rods ( $10 \mathrm{~m}$ height), the critical radius and the Rizk's equation estimate similar striking distances, but as the height of the rod increases the differences become significantly larger. This is possibly because of the fact that when the rod height is increased, the geometry of an isolated rod and the downward leader start deviating from that of the configurations used in the laboratory. This can be clearly seen for the case of the critical radius concept whose estimations are largely affected by the chosen critical radius and deliberately increase with increasing rod height. Moreover, the Rizk's equation leads to shorter striking distances than the present dynamic leader inception model. This result could be explained by the fact that the rate of increase of the electric field experienced by an upward connecting leader does not keep its tip potential constant as it propagates, contradicting the main assumption considered by Rizk [5,6].

On the other hand, the striking distances computed with the Petrov and Waters' model $[15,16]$ are even shorter than in the case of static leader inception. It is because the critical rate of change of the induced potential at the rod's tip used in [16] as criterion is much larger than in the real case of upward connecting leaders. Moreover, neither the length of the streamers nor the rate of change of the induced rod potential seem to be adequate parameters to evaluate the inception of upward connecting leaders. As can be seen in figures 3(a) and $4(a)$, the extension of the streamer zone largely changes for different cases. For all the calculations performed in this paper, the extension of the corona streamer zone at the moment of unstable leader inception varied between 0.7 and $2 \mathrm{~m}$. Furthermore, the rate of change of the background electric field required to compensate the potential drop in a newly formed leader segment during the propagation of the leader depends upon its velocity and leader channel potential gradient. These parameters are also observed to significantly change for different conditions during the inception and propagation of upward connecting leaders [28].

\section{Conclusions}

The leader inception condition from grounded isolated rods is computed by considering the changing background electric field produced by a descending downward leader as well as the injected charge by streamers and aborted leaders. The leader inception model is validated by comparing the model simulations with long gap experiments and with an altitude triggered lightning experiment. It is predicted that there is an 'optimum' tip radius which improves the lightning efficiency of a rod, in agreement with the field observations of competing rods where only blunt rods with tip diameter ranging from 12.7 to $25.4 \mathrm{~mm}$ were struck by lightning $[22,23]$. However, these rods are found to only slightly maximize the striking distance. In general, the computed differences on the height of the downward leader required to incept a stable upward leader from $10 \mathrm{~m}$ height rods with tip radii ranging from 2 to $400 \mathrm{~mm}$ are found to be lower than about $10 \%$. Moreover, these differences between sharp and blunt rods become even less significant as the height of the rod increases. Nonetheless, as seen from the 
above experiment $[22,23]$, this $10 \%$ difference can produce a large effect on the results of field observations, depending upon the placement of the competing rods. In addition, the obtained results clearly suggest that the temporal change of the background electric field, given mainly by the downward leader velocity, has to be taken into account in evaluating the leader inception from grounded rods. The conditions required to initiate a stable upward leader from an isolated rod for the extreme measured values of the downward leader velocity of $8 \times 10^{4}$ and $3.9 \times 10^{6} \mathrm{~m} \mathrm{~s}^{-1}$ differ by more than $80 \%$. Besides, the striking distances computed with the present dynamic leader inception model are significantly larger than the ones statically estimated considering an invariant background field.

\section{Acknowledgments}

The research work reported here is funded partly by the Swedish Research Council (grant no G-EG/GU 1448-306), the Swedish Rescue Services Agency (grant no 012-70392003) and a donation to Uppsala University by John and Svea Andersson.

\section{References}

[1] Les Renardières Group 1972 Research on long air gap discharges at Les Renardieres Electra 23 53-157

[2] Les Renardières Group 1974 Research on long air gap discharges-1973 results Electra 35 47-155

[3] Les Renardières Group 1977 Positive discharges in long air gaps-1975 results and conclusions Electra 53 31-132

[4] Carrara G and Thione L 1976 Switching surge strength of large air gaps: a physical approach IEEE Trans. Power Appl. Syst. PAS-95 512-24

[5] Rizk F 1989 A model for switching impulse leader inception and breakdown of long air-gaps IEEE Trans. Power Deliv. 4 596-603

[6] Rizk F 1989 Switching impulse strength of air insulation: leader inception criterion IEEE Trans. Power Deliv. PWRD-4 2187-95

[7] Bondiou A and Gallimberti I 1994 Theoretical modeling of the development of the positive spark in long gaps J. Phys. D: Appl. Phys. 27 1252-66

[8] Fofana I and Beroual A 1997 A predictive model of the positive discharge in long air gaps under pure and oscillating impulse shapes J. Phys. D: Appl. Phys. 30 1653-67

[9] Goelian N, Lalande P, Bondiou-Clergerie A, Bacchiega G L, Gazzani A and Gallimberti I 1997 A simplified model for the simulation of positive-spark development in long air gaps J. Phys. D: Appl. Phys. 30 2441-52

[10] Eriksson A J 1987 An improved electrogeometric model for transmission line shielding analysis IEEE Trans. Power Deliv. PWDR-2 871-86

[11] Dellera L and Garbagnati E 1990 Lightning strike simulation by means of the Leader Progression Model: I. Description of the model and evaluation of free-standing structures IEEE Trans. Power Deliv. PWRD-5 2009-23

[12] D'Alessandro F 2003 Striking distance factors and practical lightning rod installations: a quantitative study J. Electrostat. 59 25-41

[13] Bernardi M, Dellera L, Garbagnati E and Sartorio G 1996 Leader progression model of lightning: updating of the model on the basis of recent test results Proc. 23rd ICLP (Florence) 399-407
[14] Rizk F 1994 Modeling of lightning incidence to tall structures Part I: theory, IEEE Trans. Power Deliv. PWRD-9 162-70

[15] Petrov N I and Waters R T 1995 Determination of the striking distance of lightning to earthed structures Proc. R. Soc. A 450 589-601

[16] Petrov N I and D'Alessandro F 2002 Theoretical analysis of the processes involved in lightning attachment to earthed structures J. Phys. D: Appl. Phys. 35 1788-95

[17] Bazelyan E M and Raizer Y P 2000 Lightning Physics and Lightning Protection (Bristol: Institute of Physics)

[18] Lalande P 1996 Study of the lightning stroke conditions on a grounded structure Doctoral Thesis A publication of Office National d'Etudes et de Recherches A'erospatiales (ONERA)

[19] Becerra M and Cooray V 2006 A simplified physical model to determine the lightning upward connecting leader inception Trans. IEEE Power Deliv. 21 897-908

[20] Beasley W, Uman M and Rustan P 1982 Electric fields preceeding cloud-to-ground lightning flashes J. Geophys. Res. 87 4883-902

[21] Lalande P, Bondiou A, Laroche P, Eybert A, Berlandis J, Bador B, Bonamy A, Uman M and Rakov V 1998 Leader properties determined with triggered lightning techniques J. Geophys. Res. 103 14109-15

[22] Moore C B, Aulich G D and Rison W 2000 Measurements of lightning rod responses to nearby strikes Geophys. Res. Lett. 27 1487-90

[23] Moore C B, Rison W, Mathis J and Aulich G 2000 Lightning rod improvements J. Appl. Meterol. 39 593-609

[24] Willet J C, Davis D A and Laroche P 1999 An experimental study of positive leaders initiating rocket-triggered lightning Atmos. Res. 51 189-219

[25] Singer H, Steinbigler H and Weiss P 1974 A charge simulation method for the calculation of high voltage fields IEEE Trans. Power Appl. Syst. PAS-93 1660-8

[26] Gallimberti I 1972 The mechanism of long spark formation J. Phys. Coll. 40 193-250

[27] Aleksandrov N L, Bazelyan E M, D'Alessando F and Raizer Y P 2005 Dependence of lightning rod efficacy on its geometric dimensions-a computer simulation J. Phys. D: Appl. Phys. 38 1225-38

[28] Becerra M and Cooray V 2006 A self-consistent upward leader propagation model J. Phys. D: Appl. Phys. 39 3708-15

[29] Cooray V and Rakov V and Theethayi N 2004 The relationship between the leader charge and the return stroke current-Berger's data revisited J. Electrostatics at press

[30] Thomson E M 1985 A theoretical study of electrostatic field wave shapes from lightning leaders J. Geophys. Res. 90 8125-35

[31] Rakov V and Uman M 2003 Lightning: Physics and Effects (Cambridge: Cambridge University Press) ISBN 0521 58327

[32] Krider P, Weidman C and Noggle R 1977 The electric fields produced by lightning stepped leaders J. Geophys. Res. 82 951-60

[33] Becerra M, Cooray V and Zainal H 2005 Location of the vulnerable points to be struck by lightning in complex structures Proc. Int. Conf. Lightning and Static Electricity 2005 (ICOLSE 2005) (Seattle, USA) GND-20

[34] Becerra M, Cooray V and Roman F 2006 Striking distance of vulnerable points to be struck by lightning in complex structures Proc. 28th Int. Conf. on Lightning Protection, ICLP 2006 (Kanazawa, Japan) 608-13

[35] Aleksandrov N L, Bazelyan E M and Raizer Y P 2005 The effect of a corona discharge on a lightning attachment Plasma Phys. Rep. 31 75-91 